\title{
Enhancing Parallel Robots Accuracy with Redundant Sensors
}

\author{
Frédéric Marquet, Olivier Company, Sébastien Krut, François Pierrot \\ LIRMM - UMR 5506 CNRS - Université Montpellier 2 \\ 161 rue Ada, 34392 Montpellier Cedex 5, France \\ $<$ marquet, company, krut, pierrot >@lirmm.fr
}

\begin{abstract}
This paper introduces a control strategy based on redundant sensors that leads to parallel robots accuracy enhancement. The method is presented in general, then applied to a 4-dof parallel robot. Practical implementation issues, simulation results and experimental validation are addressed.
\end{abstract}

\section{Introduction}

After the first ideas of parallel mechanisms proposed by Gough [1] or Stewart [2], introducing the idea that an excellent stiffness could be obtained with PKM (Parallel Kinematic Machines), Clavel proposed in the late 80's the famous Delta structure [3] as a base for a "family" of parallel machines dedicated to high-speed applications. Thus many PKM have been used for pick-and-place and more recently for machining: in both application domains, PKM are considered as "more accurate" than their serial counterparts; in fact, this issue is controversial: by principle, PKM should be more accurate (because actuated joints positioning errors do not add to each others [4]), but in practice it is not always the case (because PKM involve many passive joints leading to a complex error model).

Consequently, in recent researches, a lot of effort has been dedicated to PKM calibration ([5][6]), with a very promising trend to provide self calibration techniques ([7][9]), and a continuous fundamental work for refining the mathematical tools involved in calibration ([8][10]).

Such works have dramatically improved the obtained performances regarding PKM accuracy, but few drawbacks remain: any calibration procedure is tedious, whatever the measurement systems are, and even the best calibration results are not guaranteed for the complete machine life cycle. The track which is proposed to consider here is different: improving PKM accuracy by using real-time redundant measurements.

In previous works, redundant position sensors have been mostly used as a convenient mean for solving forward kinematic problems, and the trend is to try to reduce as much as possible the number of redundant sensors needed to solve such problems ([11][12][13][14]). However, for most parallel applications, solving the Forward Kinematic problem is only a small part of the control needs, and it is often run at the user request only (to get the knowledge of the robot actual position) and not in real-time. Indeed the typical control scheme of a parallel robot is represented in Figure 1. The trajectory is firstly generated in the
Cartesian space; then each Cartesian location is transformed into a position vector in the joint space thanks to the Inverse Kinematic Model (IKM); the control (that is, the hard real-time part of the process) is finally done in that latter space; the Forward Kinematic Model (FKM) is often implemented as a HMI routine, or only runs at initialization phase.

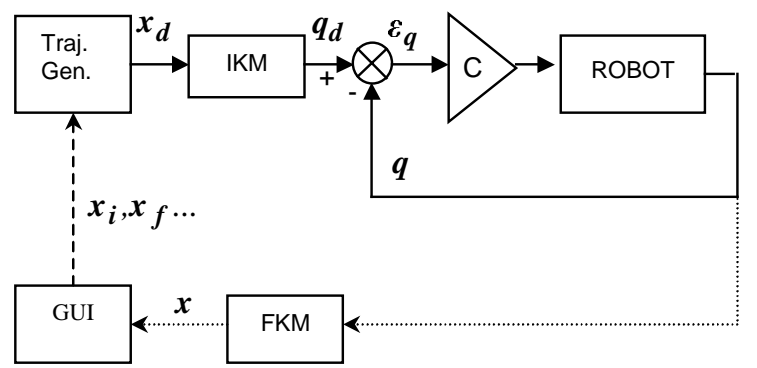

Figure 1. Typical PKM control architecture

Of course, redundant sensors can help to solve the FKM by offering closed form solutions by speeding up an iterative numerical computation; this point will be briefly recalled and addressed for our specific case.

However the point of this paper is to use one opportunity offered by PKM: they are built with many passive joints that are often ideal locations to place sensors at low additional costs. On the opposite of the traditional pointof-view, it is proposed here to use as many additional sensors as possible, and to include them in a real-time control scheme consistent with the usual solution depicted in Figure 1.

In the following sections, the proposed strategy is firstly described, then simulated, and its implementation on a specific 4-degree-of-freedom (dof) PKM is explained. Simulation and experimental results let us expect that sensor redundancy could be useful to improve the accuracy of PKM in their daily use.

\section{Use of redundant sensors data in the PKM control scheme}

Position errors occur for many reasons on a PKM:

- Control errors; basically, the actuator is not at the right position at the right time; this is measured by the usual position sensors and is supposed to be managed by controllers; 
- Kinematic model errors; for practical reasons, actual sizes and relative locations of all links and joints are not exactly as envisioned when the PKM was designed. This is not measured by the usual position sensors, and is often considered as a calibration problem, but it could be sensed by sensors placed on passive joints.

- Higher order errors; this could come from mechanical or thermal deformations, or material aging leading to increased backlash for example. This is extremely complex to model, and thus extremely complex to compensate by calibration; again, depending on sensors resolution, part of those errors could be sensed by redundant sensors.

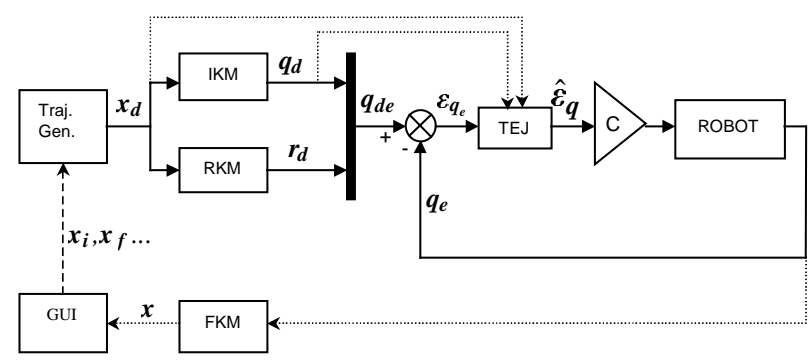

Figure 2. Architecture of the proposed control strategy

\begin{tabular}{|c|c|c|}
\hline Name & Size & Description \\
\hline$x_{d}$ & $n \times 1$ & Nacelle desired position \\
\hline$q_{d}$ & $n \times 1$ & Motors desired position \\
\hline$r_{d}$ & $r \times 1$ & Desired redundant positions \\
\hline$q_{d e}$ & $(n+r) \times 1$ & Desired extended positions \\
\hline$x$ & $n \times 1$ & Nacelle position \\
\hline$q_{e}$ & $(n+r) \times 1$ & Extended positions \\
\hline$\varepsilon_{q e}$ & $(n+r) \times 1$ & Extended errors \\
\hline$\hat{\varepsilon}_{q}$ & $n \times 1$ & Modified errors \\
\hline TEJ & $n \times(n+r)$ & $\begin{array}{l}\text { Extended to joint } \\
\text { transformation matrix } \\
\boldsymbol{M}^{+}=\boldsymbol{M}^{+}\left(\boldsymbol{x}_{\boldsymbol{d}}, \boldsymbol{q}_{\boldsymbol{d}}\right)\end{array}$ \\
\hline
\end{tabular}

Table 1. Variables definition

In the proposed strategy (Figure 2), the Cartesian Trajectory Generation module (that is the desired Cartesian location) feed two kinematic models:

- The classical Inverse Kinematic Model giving the set of actuated joint positions; for a $n$-motor robot, the IKM provides a $n \times l$ vector;

- A Redundant Kinematic Model (RKM), which gives the set of corresponding redundant positions; if $r$ redundant measurements are available, the RKM provides a $r \times 1$ vector (Table 1). As stated above, the easiest way to get redundant measurements is to install position sensors on some of the robot passive joints; indeed, those redundant measurements can be done by external means as well (e.g.: direct measure of the PKM nacelle position along one direction with a telemeter, ...)

Both position vectors are then grouped to form a single $(n+r) \mathrm{x} l$ vector, called the vector of extended positions. This vector is the controlled variable, and is compared to a corresponding vector of extended sensor measurement. Different solutions could be used to go from the $(n+r) \mathrm{x} 1$ error vector to the $n \times 1$ command vector to be sent to the motors amplifiers. It is proposed here to derive a matrix mapping the extended error vector into a vector of modified actuated joint errors; then classical controllers could be applied.

Obviously, it is still possible to compute the FKM when required by the robot user.

In the following sections, a practical implementation of the above ideas will be presented.

\section{Application to the $\mathbf{H} 4$ robot}

$\mathrm{H} 4$ is a 4-dof PKM (Figure 3) whose mechanical design belongs to the family of Delta and Hexa robots. Many passive joints are used for such mechanisms (U joints, ball joints, etc.) and depending on the technology, it could be easy or not to add redundant sensors on those passive joints.

For example, it is clear on the architectural scheme in Figure 4 that the three revolute passive joints could be easy-and-low-cost candidates for a redundant sensor.

One could also install a telemeter on the robot base to measure one distance between base and nacelle: this could give information about the position along $z$ axis.

It has been decided to place only one redundant sensor on the pivot joint on which the tool is mounted.

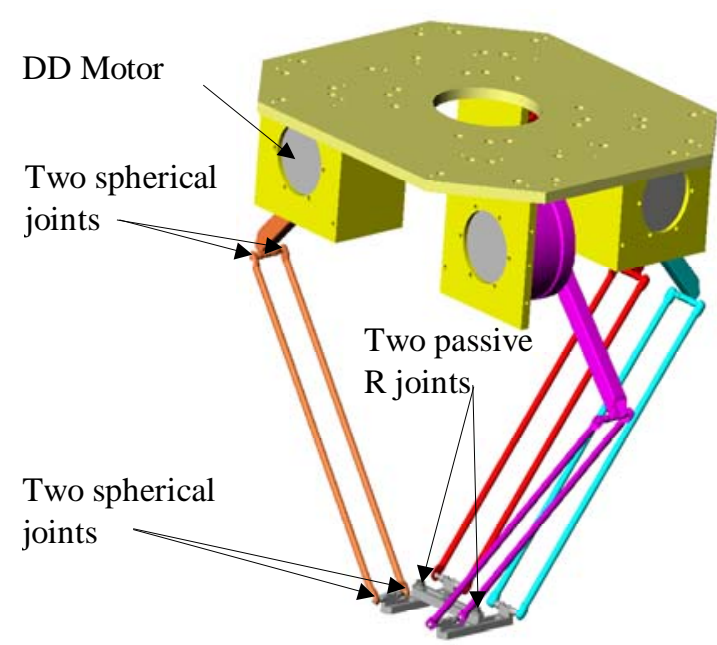

Figure 3. CAD model of the first $\mathrm{H} 4$ prototype 


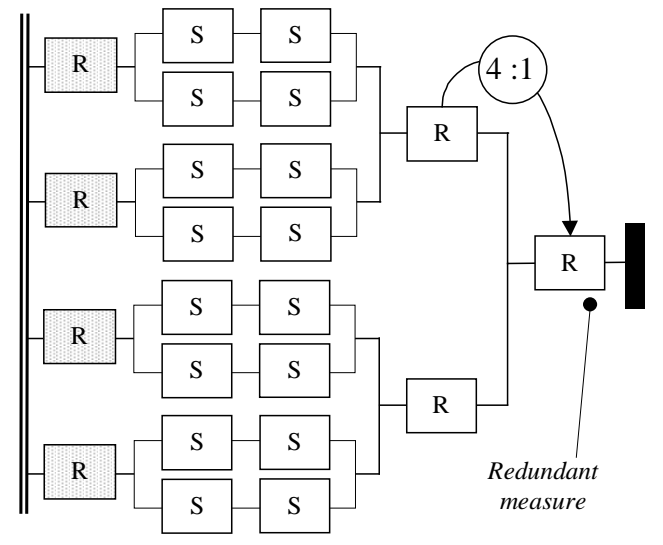

Figure 4. Architectural scheme of the $\mathbf{H 4}$ prototype

At this stage, the IKM, RKM and TJE relations have to be derived.

\section{- IKM}

In this section the relation between actuators and nacelle position represented by, respectively, $\boldsymbol{q}=\left[q_{1}, q_{2}, q_{3}, q_{4}\right]^{T}$ and $\boldsymbol{x}=[x, y, z, \theta]^{T}$ is derived.

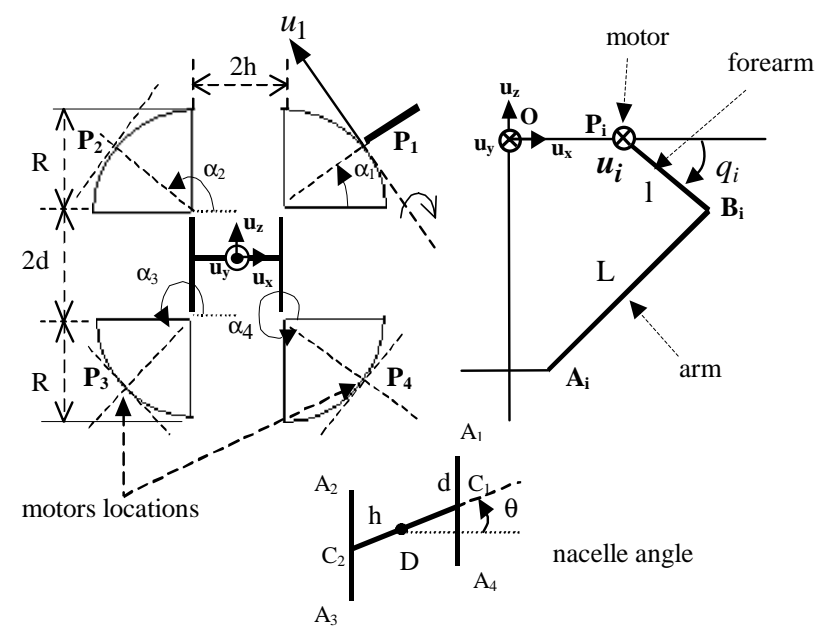

Figure 5. Design parameters

The selected design is described in Figure 5, where the following parameters have been chosen:

$$
\begin{aligned}
& \alpha_{1}=0, \quad \alpha_{2}=\pi, \quad \alpha_{3}=3 \pi / 2 \quad \alpha_{4}=3 \pi / 2 \\
& \boldsymbol{u}_{\boldsymbol{1}}=\boldsymbol{u}_{\boldsymbol{y}}, \quad \boldsymbol{u}_{\mathbf{2}}=-\boldsymbol{u}_{\boldsymbol{y}}, \quad \boldsymbol{u}_{3}=\boldsymbol{u}_{\boldsymbol{x}}, \quad \boldsymbol{u}_{\boldsymbol{4}}=\boldsymbol{u}_{\boldsymbol{x}}
\end{aligned}
$$

As it is usual for most parallel robots, the inverse position relations is easy to derive. If $\boldsymbol{v}_{\boldsymbol{i}}$ is the vector corresponding to the $i^{\text {th }}$ arm (vector from $\mathbf{A}_{\mathbf{i}}$ to $\mathbf{B}_{\mathbf{i}}$ ), it can be written that its norm is constant and equal to $\mathrm{L}$ :

$$
\forall i \in\{1, \ldots, 4\}, \quad\left\|v_{i}\right\|^{2}=L^{2}
$$

And if $\boldsymbol{w}_{\boldsymbol{i}}$ is the vector from $\boldsymbol{P}_{\boldsymbol{i}}$ to $\boldsymbol{A}_{\boldsymbol{i}}$, it has been demonstrated that this equation leads to:

$$
M_{i} \cos q_{i}+N_{i} \sin q_{i}=G_{i}
$$

where:

$$
\begin{aligned}
& M_{i}=-2 l\left(\left(\boldsymbol{w}_{\boldsymbol{i}} \bullet \boldsymbol{u}_{\boldsymbol{x}}\right) \cos \alpha_{i}+\left(\boldsymbol{w}_{\boldsymbol{i}} \bullet \boldsymbol{u}_{\boldsymbol{y}}\right) \sin \alpha_{i}\right) \\
& N_{i}=2 l\left(\boldsymbol{w}_{\boldsymbol{i}} \bullet \boldsymbol{u}_{z}\right) \\
& G_{i}=L^{2}-l^{2}-\left\|\boldsymbol{w}_{\boldsymbol{i}}\right\|^{\mathbf{2}}
\end{aligned}
$$

Resorting to the following new variable:

$$
t_{i}=\tan \frac{\theta_{i}}{2}
$$

actuators positions are given by:

$$
q_{i}=2 \tan ^{-1}\left(\frac{-b_{i} \pm \sqrt{b_{i}^{2}-4 a_{i} c_{i}}}{2 a_{i}}\right)
$$

where :

$$
\begin{aligned}
& a_{i}=G_{i}+M_{i} \\
& b_{i}=-2 N_{i} \\
& c_{i}=G_{i}-M_{i}
\end{aligned}
$$

Using this way of solving equation (1) a mathematical singularity can occur when $a_{i}=0$. It is possible to avoid this problem by introducing the following new variables:

$$
\tan \alpha_{i}=\frac{N_{i}}{M_{i}} \quad \cos \beta_{i}=\frac{G_{i}}{M_{i}}
$$

Then the expression of $q_{i}$ becomes:

$$
q i=\tan ^{-1} \frac{N_{i}}{M_{i}} \pm \cos ^{-1} \frac{G_{i}}{\sqrt{M_{i}^{2}+N_{i}^{2}}}
$$

\section{- $\quad$ RKM}

The set of redundant positions is here trivial to determine:

$$
\boldsymbol{r}=[4 \theta]
$$

Note that the multiplying factor (4) comes from the mechanical amplification system installed between nacelle and gripper axis (Figure 6).

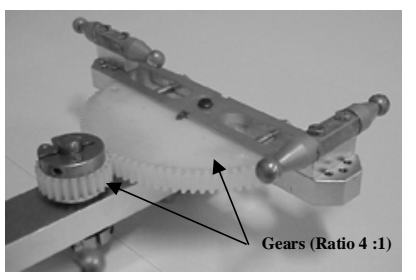

Figure 6. Amplification system 


\section{- $\quad$ TEJ}

The first step to determine TEJ is to establish the relation between actuators and nacelle velocity respectively represented by $\dot{\boldsymbol{q}}$ and $\dot{\boldsymbol{x}}$. As for many parallel robots, this expression is derived as follows:

$$
J_{x} \dot{x}=J_{q} \dot{q}
$$

To derive equation (2), the following classical property that relates the velocities $\boldsymbol{V}_{A_{i}}$ and $\boldsymbol{V}_{B_{i}}$ of the two points $\boldsymbol{A}_{\boldsymbol{i}}$ and $\boldsymbol{B}_{\boldsymbol{i}}$ is used:

$$
\boldsymbol{V}_{A_{i}} \bullet v_{i}=V_{B_{i}} \bullet v_{i}
$$

If $\boldsymbol{n}_{1}$ and $\boldsymbol{n}_{2}$ are the vectors from $\boldsymbol{D}$ to $\boldsymbol{C}_{\boldsymbol{1}}$ and $\boldsymbol{C}_{2}$ respectively and $\boldsymbol{p}_{\boldsymbol{i}}$ the vector from $\boldsymbol{P}_{\boldsymbol{i}}$ to $\boldsymbol{B}_{\boldsymbol{i}}$, the application of equation (3) to the four robot arms leads to:

$$
J_{x}=\left[\begin{array}{llll}
v_{1} \bullet u_{x} & v_{1} \bullet u_{y} & v_{1} \bullet u_{z} & \left(n_{1} \times v_{1}\right) \bullet u_{z} \\
v_{2} \bullet u_{x} & v_{2} \bullet u_{y} & v_{2} \bullet u_{z} & \left(n_{2} \times v_{2}\right) \bullet u_{z} \\
v_{3} \bullet u_{x} & v_{3} \bullet u_{y} & v_{3} \bullet u_{z} & \left(n_{2} \times v_{3}\right) \bullet u_{z} \\
v_{4} \bullet u_{x} & v_{4} \bullet u_{y} & v_{4} \bullet u_{z} & \left(n_{1} \times v_{4}\right) \bullet u_{z}
\end{array}\right]
$$

$$
J_{q}=\left[\begin{array}{cccc}
\left(p_{1} \times v_{1}\right) \bullet u_{y} & 0 & 0 & 0 \\
0 & \left(p_{2} \times v_{2}\right) \bullet-u_{y} & 0 & 0 \\
0 & 0 & \left(p_{3} \times v_{3}\right) \bullet u_{x} & 0 \\
0 & 0 & 0 & \left(p_{4} \times v_{4}\right) \bullet u_{x}
\end{array}\right]
$$

and, if the mechanism is not in a singular configuration the jacobian matrix is:

$$
J=J_{x}^{-1} J_{q}
$$

Thus, away from singular positions, a small variation $\Delta \theta$ on $\theta$ is related to small variations on the actuated variables, noted $\Delta q_{1}, \cdots, \Delta q_{4}$, by the expression:

$$
\Delta \theta=J_{4,1} \Delta q_{1}+J_{4,2} \Delta q_{2}+J_{4,3} \Delta q_{3}+J_{4,4} \Delta q_{4}
$$

The relation between extended errors and joint errors is then:

$$
M \Lambda q=\Lambda q_{e}
$$

where:

$$
\begin{gathered}
\Delta \boldsymbol{q}_{e}=\left[\Delta q_{1} ; \Delta q_{2} ; \Delta q_{3} ; \Delta q_{4} ; \Delta \theta\right]^{T} \\
\boldsymbol{\Delta q}=\left[\Delta q_{1} ; \Delta q_{2} ; \Delta q_{3} ; \Delta q_{4}\right]^{T}
\end{gathered}
$$

$$
\boldsymbol{M}=\left[\begin{array}{cccc}
1 & 0 & 0 & 0 \\
0 & 1 & 0 & 0 \\
0 & 0 & 1 & 0 \\
0 & 0 & 0 & 1 \\
\boldsymbol{J}_{4,1} & \boldsymbol{J}_{4,2} & \boldsymbol{J}_{4,3} & \boldsymbol{J}_{4,4}
\end{array}\right] \in \mathfrak{R}^{5 \times 4}
$$

This linear over-determined system has to be inverted to get the sought relationship that gives the modified error vector, $\boldsymbol{\Lambda} \hat{\boldsymbol{q}}$. A convenient way to do so is to rely on pseudo-inversion that gives the least square better solution:

$$
\boldsymbol{\Lambda q}=M^{+} \Delta q_{e}
$$

\section{Implementation and results}

This section presents practical implementation issues, simulation and experimental results.

\section{Practical implementation:}

Regarding practical implementation, two issues have been addressed:

- adding the redundant sensor; an optical encoder (resolution: 14400 tops/rev) mounted on the gripper rotation axis has been selected (Figure 7, Figure 8, Figure 9); it gives a $14400 * 4$ tops/rev resolution at nacelle level;

- installing measurement facilities; a 3D vision-based measurement system (SAGEIS SM3D) has been selected; this system uses 3 linear CCD fixed on the ground (Figure 10) and a set of 4 LED mounted on the nacelle; a calibration process has been carried out, but is not described here (the process followed is similar to the one prescribed in Figure 7).

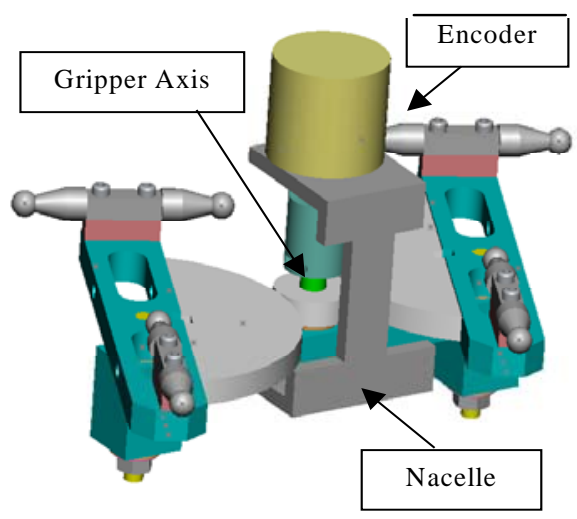

Figure 7. The encoder is mounted on the gripper axis 


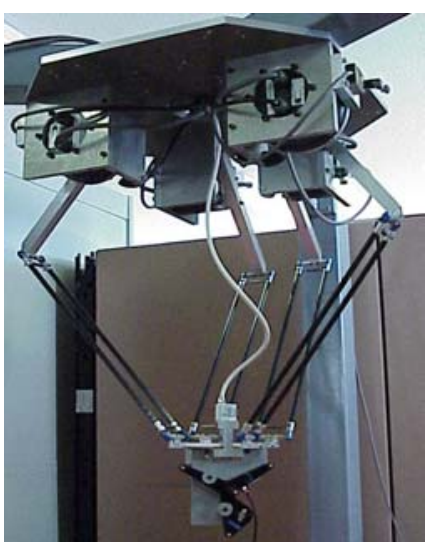

Figure 8. General view of $\mathrm{H} 4$ equipped with a redundant sensor, and a 3D measurement system

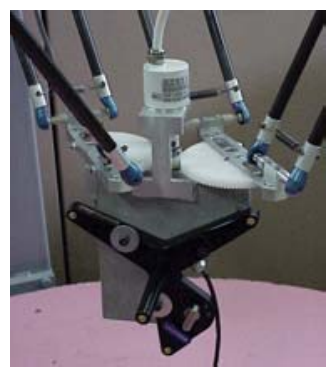

Figure 9. Closer view of the encoder and the 4-LED based measurement system

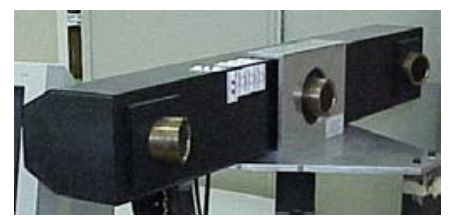

Figure 10. A view of the SAGEIS SM3D vision system with 3 linear CCD.

\section{- Simulation results}

A Matlab simulation platform has been implemented to compare the classical joint control to the "redundant sensor control" using the additional measurement of nacelle angle. Robot behavior is represented by the dynamic model described in [16]. Errors on robot geometrical parameters are introduced, as arms and forearms lengths, motors locations, nacelle sizes:

- errors on arms length $L_{i}$ from $-3 \mathrm{~mm}$ to $10 \mathrm{~mm}$,

- errors on fore arms length $l_{i}$ from $-0.4 \mathrm{~mm}$ to $0.6 \mathrm{~mm}$,

- errors on radius $R_{i}$ from $-0.5 \mathrm{~mm}$ to $0.5 \mathrm{~mm}$,

- errors on $d_{i}$ from -0.1 to $0.1 \mathrm{~mm}$.

A $0.2 \mathrm{~s}$ displacement from $\boldsymbol{x}_{i}=[0 \mathrm{~mm} ; 0 \mathrm{~mm} ;-500 \mathrm{~mm} ; 0$ $\mathrm{rad}]$ to $\boldsymbol{x}_{f}=[100 \mathrm{~mm} ;-100 \mathrm{~mm} ;-400 \mathrm{~mm} ; 0.2 \mathrm{rad}]$ was then simulated in the case of:

- a PD joint controller and no error on geometrical parameters (Figure 11),
- a PD joint controller in presence of errors on geometrical parameters (Figure 12),

- a controller using the redundant sensor information in presence of errors on geometrical parameters (Figure 13).

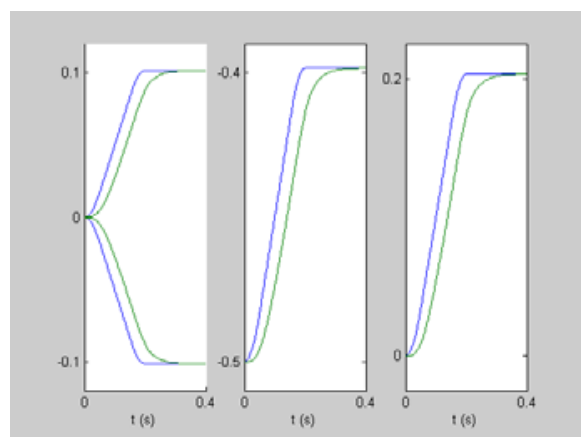

Figure 11. Control using a simple PD joint controller (ideal case)

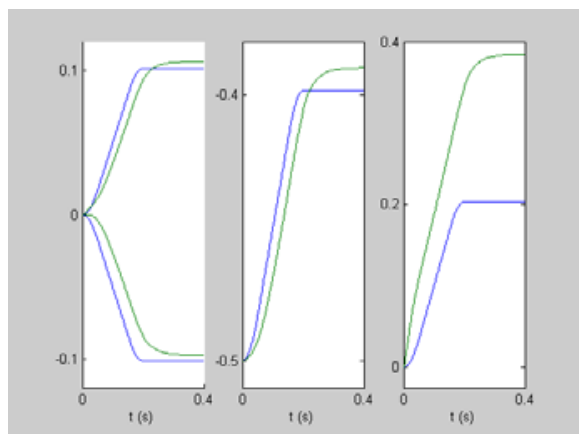

Figure 12. Control using a PD joint controller in the case of errors on geometrical parameters

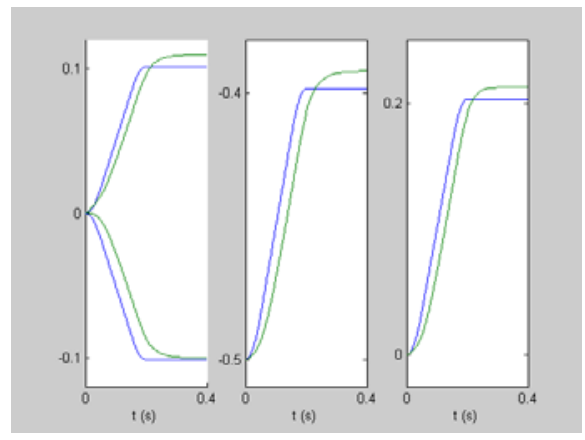

Figure 13. Control using the redundant information on theta

As shown in Figure 11, when there is no error on geometrical parameters, a PD joint controller permits to obtain good results (no static error) whereas it can't ensure a proper control if errors are introduced in the model representing the "real" robot (Figure 12): a large static error remains in Cartesian space (i.e.: the actuated joints are at their desired positions, while the end-effector location is not correct). The interest of the control using a sensor measuring nacelle angle is then clearly 
demonstrated in Figure 13: static errors remind the same on $\mathrm{x}, \mathrm{y}$ and $\mathrm{z}$, but the static error on $\theta$ is lower.

\section{Experimental results}

The control of $\mathrm{H} 4$ robot is realized by a simple PC (Windows NT, Pentium II $200 \mathrm{MHz}$ ) and RTX (Real Time eXtension) is used has real time software to ensure the control task periodicity (see [16] for more information regarding $\mathrm{H} 4$ control). In order to compare the results obtained with and without the redundant sensor, a set of Cartesian positions is obtained by scanning 625 locations throughout the $\mathrm{H} 4$ workspace (see Table 2).

\begin{tabular}{|c|c|c|c|}
\hline & Min. value & Max. value & Step size \\
\hline $\mathrm{x}(\mathrm{mm})$ & -100 & 100 & 50 \\
\hline $\mathrm{y}(\mathrm{mm})$ & -100 & 100 & 50 \\
\hline $\mathrm{z}(\mathrm{mm})$ & -450 & -350 & 25 \\
\hline$\theta(\mathrm{rad})$ & $(-\pi / 6) * 4$ & $(\pi / 6) * 4$ & $(\pi / 12) * 4$ \\
\hline
\end{tabular}

Table 2. Locations for errors measurement

For each location, encoders give motors positions and an estimated nacelle position is obtained by computing the FKM. This estimated position is then compared to the one given by the $3 \mathrm{D}$ vision based measurement system. The matrix $\boldsymbol{M}=\boldsymbol{M}(\boldsymbol{x}, \boldsymbol{q})$ that permits to compute the vector of modified errors is evaluated for $\boldsymbol{x}=\boldsymbol{x}_{\boldsymbol{d}}$ and $\boldsymbol{q}=\boldsymbol{q}_{\boldsymbol{d}}$ for not using the iterative FKM.

Table 3 sums up the experimental results obtained for a classical joint control where a usual controller is implemented (left value) and when using the redundant measure of nacelle angle (right value):

\begin{tabular}{|c|c|c|c|c|c|c|c|c|}
\hline & \multicolumn{2}{|c|}{$\begin{array}{c}\text { Average } \\
\text { Errors } \\
\text { (mm or deg) }\end{array}$} & \multicolumn{2}{|c|}{$\begin{array}{l}\text { Peak to Peak } \\
\text { Errors } \\
\text { (mm or deg) }\end{array}$} & \multicolumn{2}{|c|}{$\begin{array}{c}\text { Sum of Quadratic } \\
\text { Errors } \\
\left(\mathrm{mm}^{2} / \mathrm{deg}^{2}\right) \\
\end{array}$} & \multicolumn{2}{|c|}{$\begin{array}{l}\text { Average of } \\
\text { Absolute Errors } \\
\text { (mm or deg) }\end{array}$} \\
\hline & Usual & Red. & Usual & Red. & Usual & Red. & Usual & Red. \\
\hline Err. $x$ & 2.4 & 2.6 & 14.1 & 15.3 & 10300 & 11700 & 3.3 & 3.6 \\
\hline Err. y & -6.4 & -5.7 & 12.2 & 13.0 & 29000 & 23100 & 6.4 & 5.7 \\
\hline Err. $z$ & -0.9 & -1.1 & 18.2 & 16.3 & 13100 & 10900 & 3.9 & 3.5 \\
\hline Err. $\theta$ & -0.32 & 0.02 & 3.31 & 2.73 & 231 & 128 & 0.5 & 0.3 \\
\hline $\operatorname{Norm}(x, y, z)$ & 8.8 & 8.2 & 11.7 & 11.4 & 52400 & 45700 & & \\
\hline
\end{tabular}

\section{Table 3. Sum-up of the experimental results}

These results clearly demonstrate an improvement of accuracy for the rotation whereas no change for the others dof $(x, y, z)$. The angle bias (i.e. the average value of the error) is nearly zero $(0.02 \mathrm{deg}$.) in the case of use of the redundant sensor. Moreover, in the same time, the quadratic error is reduced by $45 \%$ (231 vs. $\left.128 \mathrm{~mm}^{2}\right)$. An important accuracy improvement on theta is then realized, but not to the detriment of errors along $x, y$ and $z$ as one could has thought. This proves that other additional sensors could also be added on robot passive joints and it would increase the global accuracy of the robot.

\section{Conclusion}

This paper presented a control strategy based on the use of sensor redundancy for accuracy improvement. This method has clearly the advantage of its implantation simplicity and its low cost (only supplementary sensors are necessary). The first results obtained in the case of the H4 robot demonstrated its validity with an increase of accuracy when applying a standard joint space control (it has to be noticed that it could be also possible to obtain even better results by implementing a Cartesian controller using robot redundant FKM).

\section{References}

[1] Gough V.E., Contribution to discussion of papers on research in automotive stability, control and tyre performance. Proc. Auto Div. Inst. Mechanical Engineers, 1956-1957.

[2] Stewart D., A platform with 6 degrees of freedom. Proc. of the Inst. of Mech. engineers, 180(Part 1, 15), pp. 371-386, 1965.

[3] Clavel R., Une nouvelle structure de manipulateur parallèle pour la robotique légère, APII, 23(6), pp. 501-519, 1989.

[4] Merlet J.-P., Les robots parallèles, $2^{\text {nd }}$ Edition, Hermes, 1997.

[5] Nahvi A., Hollerbach J.M., Hayward V., Calibration of a parallel robot using multiple kinematics closed loops. In IEEE Int. Conf. on Robotics and Automation, pp. 407-412, San Diego, 8-13 May 1994

[6] Lintott A.B. et Dunlop G.R., Parallel topology robot calibration. Robotica, 15(4):395-398, 1997

[7] P. Maurine, M. Uchiyama, K. Abe, A Fully-Autonomous Procedure for Kinematic Calibration of HEXA Parallel Robots, China-Japan Bilateral Symp. On Adv. Manufacturing Eng., China, pp. 161-166, Oct. 1998

[8] Zhuang H., Yan J., et Masory O., Calibration of Stewart platforms and other parallel manipulators by minimizing inverse kinematic residuals. J. of Robotic Systems, 15(7):395-405, 1998.

[9] Khalil W. et Besnard S., Self calibration of Stewart-Gough parallel robot without extra sensors, IEEE Trans. on Robotics and Automation, 15(6):1116-1121, 1999

[10] Daney D. et Emiris I.Z., Variable elimination for reliable parallel robot calibration. In F.C. Park C.C. Iurascu, editor, Computational Kinematics, pages 133-144. 20-22 May 2001

[11] K. Cheok, J. Overholt, R. Beck, "Exact methods for determining the kinematics of a Stewart platform using additional displacement sensors", Journal of Robotics Systems, Vol. 10, No 5, pp. 6689-707, 1993

[12] L. Baron, J. Angeles, "The isotropic decoupling of the direct kinematics of parallel manipulators under sensor redundancy”, Proc. of the IEEE ICR\&A, pp. 1541-1546, 1995.

[13] I. Bonev, J. Tyu, N-J. Kim, S-K. Lee, "A simple new closedform solution of the direct kinematics of parallel manipulators using three linear extra sensors", Proc. of the IEEE ICR\&A, Atlanta, GA, pp.526-530, September 1999.

[14] V. Parenti-Castelli, R. Di Gregorio, "Determination of the actual configuration of the general Stewart platform using only one additional sensor", ASME Journal of Mechanical Design, Vol. 121, pp. 21-25, March 1999.

[15] Company O. and Pierrot F., A new 3T-1R parallel robot, ICAR '99, Tokyo, Japan, October 25-27, 1999, pp. 557-562.

[16] Hollerbach, J.M. and Wampler, C.W., The calibration index and taxonomy for robot kinematic calibration methods. The International Journal of Robotics Research, 15(6):573-591, December 1996.

[17] Pierrot F., Marquet F., Company O., Gil T., H4 parallel robot: modeling, design and preliminary experiments. IEEE Int. Conf. On Robotics and Automation, Seoul, Korea, May 2001

Acknowledgements: These researches have been partially supported by European Grant GRD1-1999-10515 “MACH21”. 